\title{
Simulation Study on Chemical Protection of a Certain Type of Transport Vehicle
}

\author{
Xuezheng Zhu ${ }^{1}$, Kunlin Nie ${ }^{1}$, Yong Tian ${ }^{2}$, Zijing $\mathrm{Ma}^{3}$, Xiaocong Nie ${ }^{1}$, Zhijie Wang ${ }^{1}$ \\ ${ }^{1}$ Insititute of NBC Defence, Beijing 102205, China \\ ${ }^{2}$ Institute of Engineering Thermophysics, Chinese Academy of Sciences, Beijing 100190, China \\ ${ }^{3}$ Ministry of Security of Northern Theater Command Army, Jinan 250002, China \\ jinlin1371780@163.com,542112706@qq.com
}

Keywords: transport vehicle; chemical contamination; protection; simulation

\begin{abstract}
With the Fluent module of ANSYS software, simulation is conducted on the protection in contaminated areas for a certain type of transport vehicle encountering chemical attack. The distribution of contaminants in the vehicle is grasped, and the contamination of typical parts is analyzed to providence evidence for protection decisions.

A certain type of transport vehicle is used for combat, carry, and transportation with high speed and strong off-road performance. In the combat operations, there exists the threat of a chemical attack on a certain type of transport vehicle. Based on the application background, the protection of the contaminated area is simulated to provide a basis for protection decisions.
\end{abstract}

\section{Basic principle of chemical protection of a certain type of transport vehicle}

The chemical protection system of transport vehicle consists of two parts, the collective protection system and the individual protective equipment. The collective protection system filters the pollutants in the air taken by dedusting booster fan with the filter absorber, and provides clean air for the vehicle, and form a certain positive pressure to resist outside pollutants into the vehicle. When the transport vehicle encounters chemical attack, the collective protection system is used to organize protection. The specific process is shown in Figure 1. When the person in the vehicle wants to operate outside the vehicle, or the collective protection system is ineffective because of the poor leakproofness of vehicle, the person wears individual protective equipment to avoid chemical hazards.

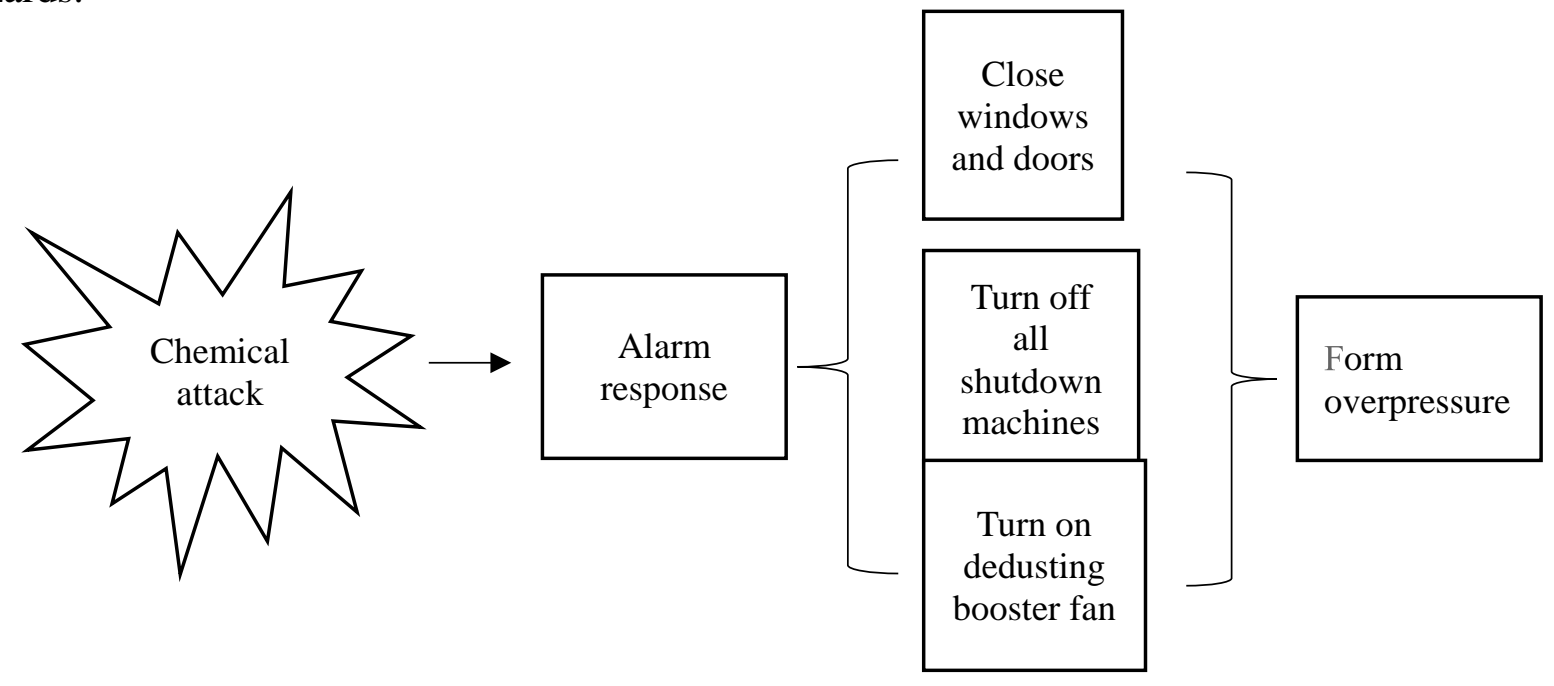

Figure 1

\section{Computational simulation of chemical protection of a certain type of transport vehicle}

In order to understand the survivability of a certain type of transport vehicle in environment with 
chemical threat and study protective measures, Fluent software is applied in this paper to simulate the process of transport vehicle crossing a chemical contaminated zone, grasp the law of pollutant diffusion, provide reference for protection decision-making, and lay the foundation for the field test.

\subsection{Set simulation environment}

Based on the operating environment and realistic simulation conditions of a certain type of transport vehicle, suppose that the vehicle encounters a chemical attack at a constant speed in a certain area (the driver window is open), the pollutants are uniformly distributed in the affected area after the attack, the chemical alarm response time of the vehicle is $7 \mathrm{~s}$. The response time of the shutdown machine is negligible, and the collective protection system runs immediately after the window is closed. Contaminants continuously penetrate into the vehicle through the gap of the driver's window (gap exists in the vehicle due to manufacturing process, and assumed concentrated in the driver window uniformly), and there is a certain distribution in the vehicle over time.

\subsection{Geometric modeling}

NX 10.0 is used to create the geometric model, as shown in Figure 2. The computational domain contains the flow field around vehicle and internal flow field of transport vehicle. The flow field around vehicle extends to 1 time the distance in front of the car, 2 times the height at the top, 1 time the width at both sides, and 3 times the length at the rear. The computational domain is large enough to ensure that the computational domain boundary conditions are as close as possible to the actual flow field, as shown in Figure 3.

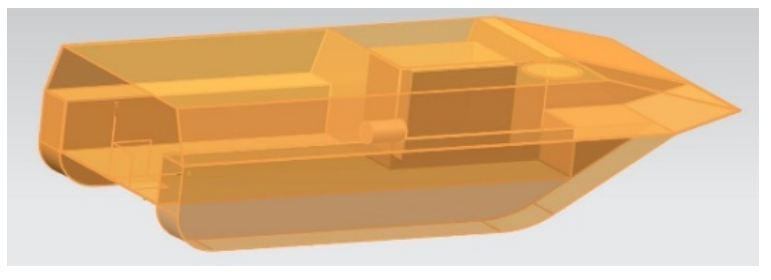

Figure 2 Geometric model of a certain type of transport vehicle

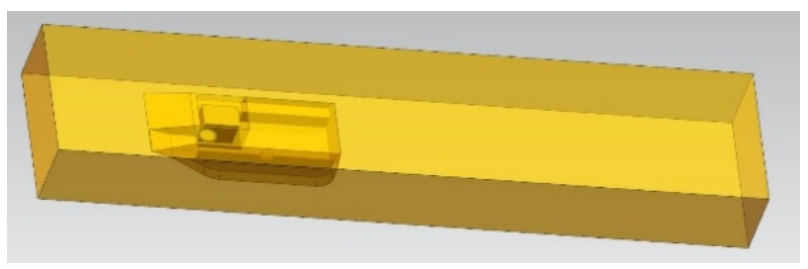

Figure 3 Simulation computational domain

\subsection{Computational grid formed}

ANSYS ICEM CFD 18.2 is used for the mesh generation of the model. All-tetrahedron unstructured grid is used, and mesh encryption is performed on key areas such as near-wall and window-hole seams. The total number of grid cells is 5536255, as shown in Figure 4.

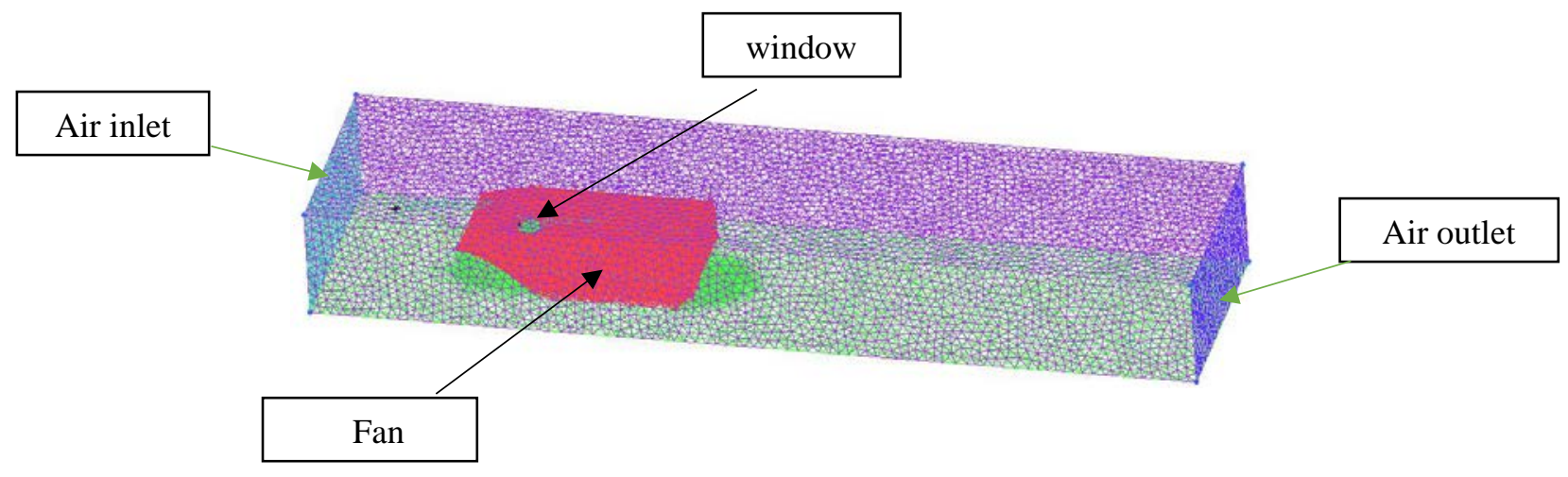

Figure 4 Simulation computational grid 


\subsection{Set computational method}

ANSYS FLUENT 18.2 is used to conduct full 3D unsteady simulation on the model.

\subsubsection{Flow model}

The simulation is based on a three-dimensional unsteady viscous compressible model, which starts the component transport equation. The turbulence model uses the Realizable $\kappa-\varepsilon$ model and the extensible wall function.

\subsubsection{Fluid model}

The computational domain fluid is regarded as binary component of air and pollutants. The physical properties of pollutants in the assumed simulation environment are shown in Table 1.

Table 1 Physical properties

\begin{tabular}{ccc}
\hline types & unit & value \\
\hline density & $\mathrm{g} / \mathrm{mL}$ & 1.1005 \\
\hline viscosity & $\mathrm{cP}$ & 1.75 \\
\hline Surface tension & $\mathrm{dyn} / \mathrm{cm}$ & 27.20 \\
\hline Diffusion coefficient & $\mathrm{cm}^{2} / \mathrm{s}$ & 0.755 \\
\hline
\end{tabular}

\subsubsection{Boundary conditions}

The computational domain boundary conditions are set as Table 2 .

Table 2 The computational domain boundary conditions

\begin{tabular}{|c|c|}
\hline position & conditions \\
\hline $\begin{array}{l}\text { The computational } \\
\text { domain inlet }\end{array}$ & $\begin{array}{l}\text { Velocity inlet: Take the vector stacking velocity of the } \\
\text { transport vehicle and wind speed, } 15 \mathrm{~m} / \mathrm{s} \text {, as the inlet flow } \\
\text { velocity; temperature: } 288 \text {; component is the air with } \\
\text { pollutants, air density: } 1295 \mathrm{mg} / \mathrm{L} \text {, pollutant concentration: } \\
0.1 \mathrm{mg} / \mathrm{L} \text {. }\end{array}$ \\
\hline $\begin{array}{l}\text { The top and both sides } \\
\text { of computational } \\
\text { domain }\end{array}$ & $\begin{array}{l}\text { As for wall boundaries, because the computational domain } \\
\text { is set large enough, free flow boundaries at the top and } \\
\text { both sides can be set as wall boundaries within a certain } \\
\text { error range, which facilitates the convergence of } \\
\text { calculations. }\end{array}$ \\
\hline $\begin{array}{c}\text { The computational } \\
\text { domain outlet }\end{array}$ & $\begin{array}{l}\text { Outlet pressure boundary: Set ambient atmosphere } \\
\text { pressure in the outlet. }\end{array}$ \\
\hline $\begin{array}{l}\text { Inner and outer surface } \\
\text { of transport vehicle }\end{array}$ & Wall boundary, viscous non-slip wall, adiabatic. \\
\hline $\begin{array}{c}\text { Window of transport } \\
\text { vehicle }\end{array}$ & $\begin{array}{l}\text { The gap is always maintained as an internal through-flow } \\
\text { surface. When the window is open, the window surface is } \\
\text { the internal through-flow surface. When the window is } \\
\text { closed, the window surface is the wall boundary. }\end{array}$ \\
\hline Fan outlet & $\begin{array}{l}\text { Before the fan is turned on, the fan outlet is set as the wall } \\
\text { boundary. After turned on, the fan outlet is set as the mass } \\
\text { flow rate inlet boundary, and the flow rate reaches } 500 \\
\mathrm{~m}^{3} / \mathrm{h} \text { after the fan is turned on. }\end{array}$ \\
\hline
\end{tabular}

\subsubsection{Initial conditions}

One important method in this paper is to obtain calculation initial field with steady calculation, (incoming pollutant concentration is 0 , window open, and fan closed), start the unsteady calculation after demining the field, including the two phases of before and after turning on fan. Incoming set is pollutant with a certain concentration before turning on fan, and the initial field is the result of 
non-polluting steady calculation mentioned before. Turn on fan, close window, and the initial field is the result of unsteady calculation mentioned before turning on fan at the last moment.

\subsubsection{Numerical solution}

The SIMPLE algorithm based on pressure-velocity coupling is used. The pressure and density are discrete with the second-order upwind scheme discrete, and momentum equation, turbulence equation, and component equation are also discrete using the second-order upwind scheme.

\subsubsection{Computation monitoring}

The unsteady calculation in this paper takes fixed time step of 0.01 second, and a total of 107 seconds of physical time is calculated. Before the fan was turned on, 7 physical seconds were calculated, that is 700 physical time steps, of which 20 steps iterative computing were performed in each physical time step. After the fan was turned on, 100 physical seconds were calculated, that is 10000 physical time steps, of which 20 steps iterative computing were performed in each physical time step. During the computation, the change of key physical quantities such as the average pressure in the vehicle, the average concentration of pollutants in the vehicle, and the concentration of pollutants at a number of key locations were monitored over time.

\subsection{The processing of computational results}

This paper focuses on the concentration distribution of pollutants in the vehicle, the change in the concentration distribution of typical locations, the impact of the fan on the concentration distribution in the vehicle, and then the impact on the personnel, when vehicles encounter chemical attack in process. Therefore, when using CFD-POST18.2 to process data, the $850 \mathrm{~mm}$ height cross section concentration distribution in the vehicle in the 3s (Figure 5), the $850 \mathrm{~mm}$ height cross section concentration distribution in the vehicle in the $7 \mathrm{~s}$ (Figure 6), and the $850 \mathrm{~mm}$ height cross section concentration distribution in the vehicle in the 7.1s (Figure 7), the average pressure change in the vehicle after the fan was turned on (Figure 8), the concentration change in the driver's position (Figure 9), and the concentration change near the position where fan contains members (Figure 10) are mainly output.

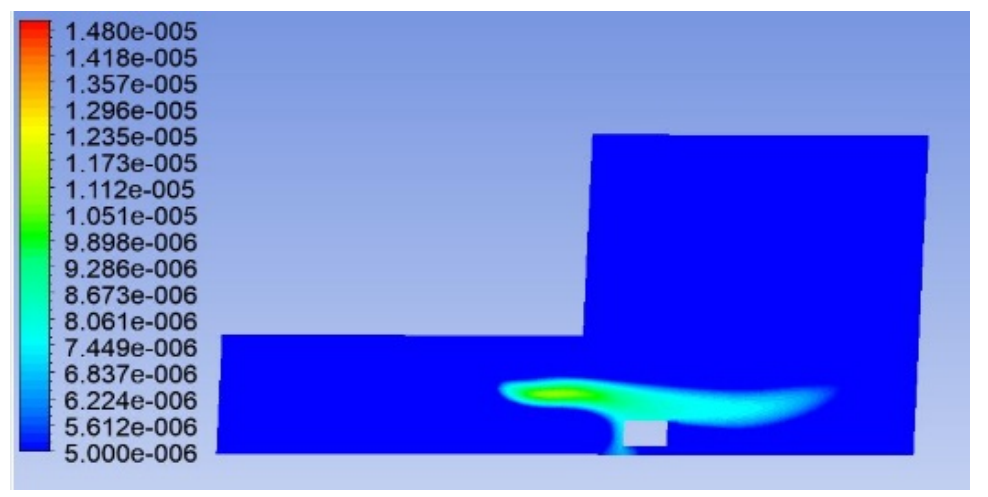

Figure 5 The $850 \mathrm{~mm}$ height cross section concentration distribution in the vehicle in the 3s

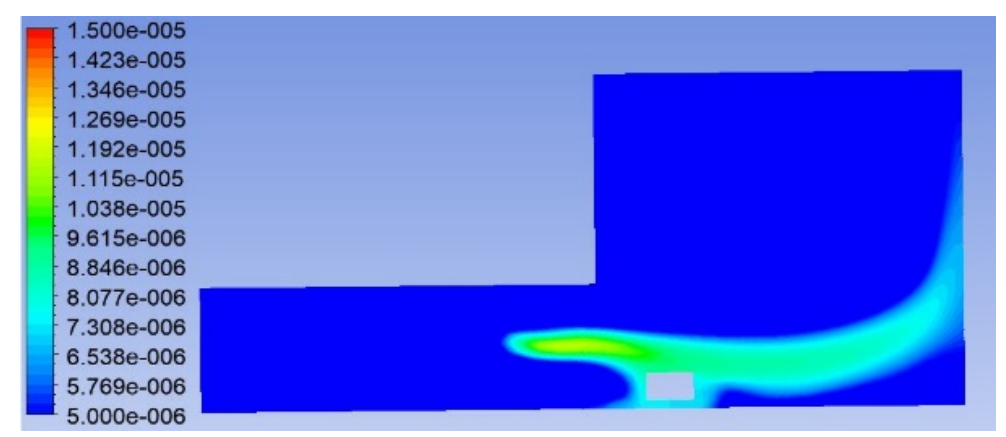

Figure 6 The 850mm height cross section concentration distribution in the vehicle in the 7s 


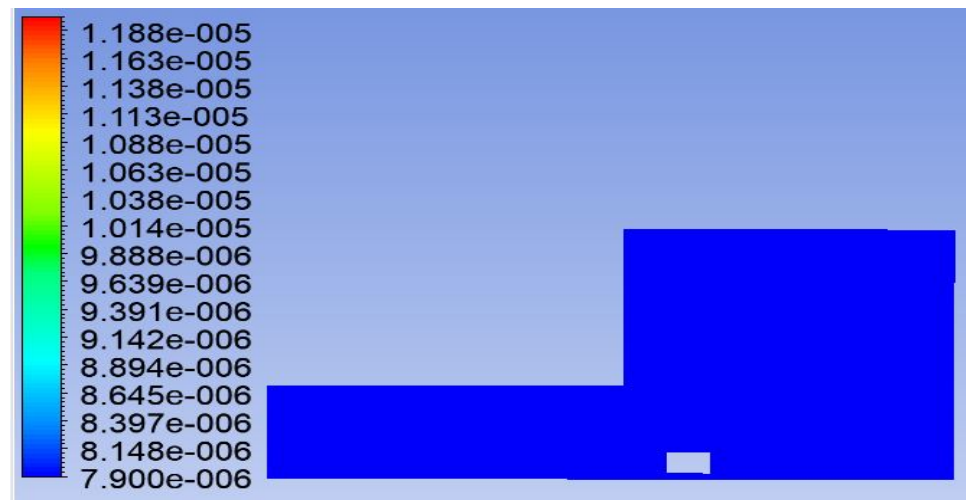

Figure 7 The $850 \mathrm{~mm}$ height cross section concentration distribution in the vehicle in the 7.1s

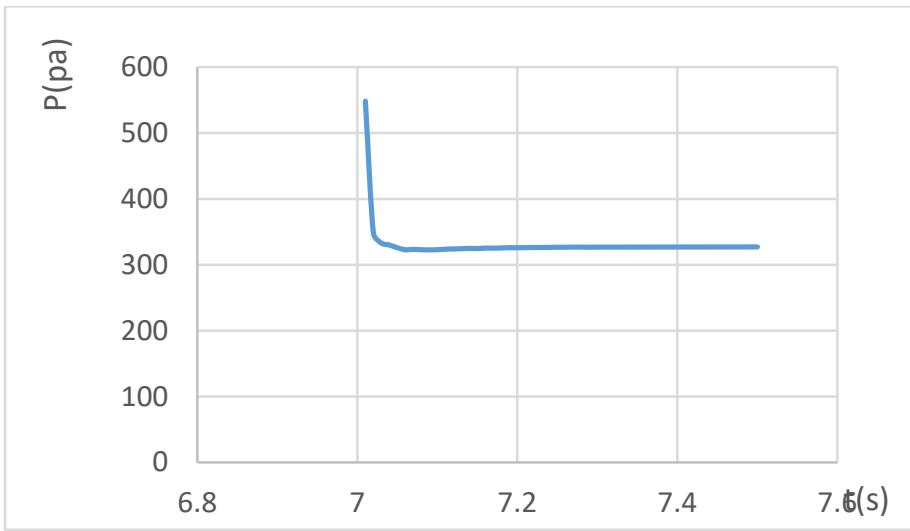

Figure 8 The average pressure change in the vehicle after the fan was turned on.

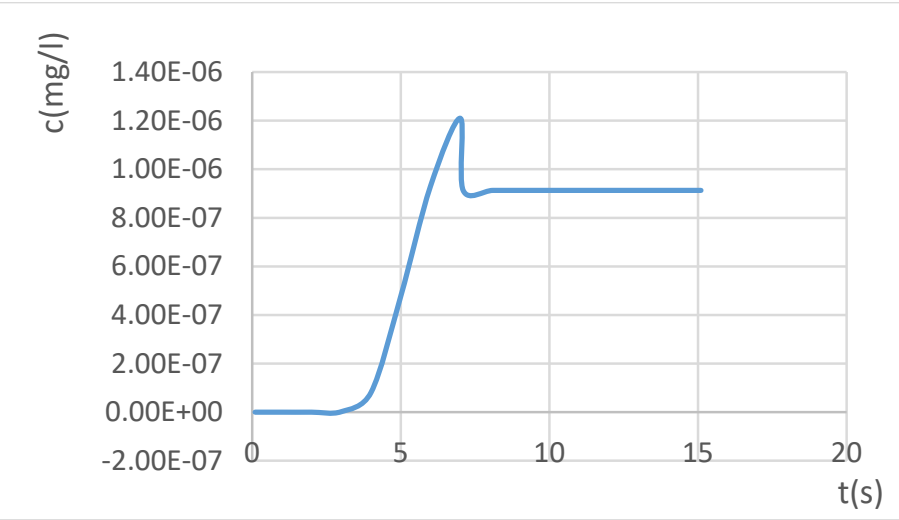

Figure 9 The concentration change in the driver's position.

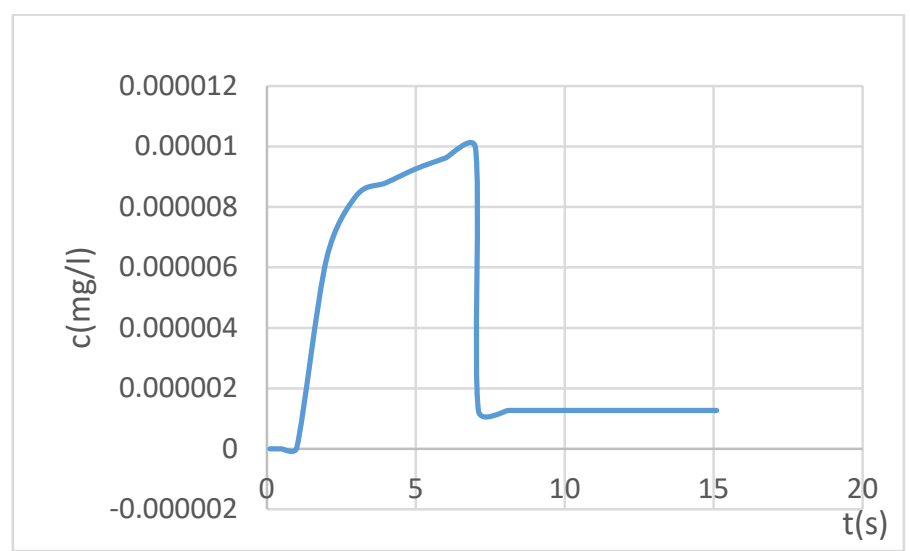

Figure 10 The concentration change near the position where fan contains members. 


\section{Preliminary suggestions on chemical protection of a certain type of transport vehicle}

As can be seen from Figure 5 and Figure 6, before the fan is turned on, pollutants infiltrate into the vehicle along the window, and gradually spread to the right rear of the vehicle body, the front concentration is higher than the rear concentration, and requirement of chemical protection in the right front passenger side of the vehicle is high. It can be seen from Figure 10 that the concentration of the personnel in the position near the fan before the fan started exceeds the safety dose, and for safety reasons, certain measure of protection can be taken when necessary and the concentration is low at the driver (Figure 9), and the driver can operate normally. It can be seen from Figure 6, Figure 7 and Figure 8 that when the vehicle is with excellent tightness, the vehicle will quickly form an overpressure to reach a steady state within a very short time (within $0.1 \mathrm{~s}$ ), the pollutants in the vehicle will be mixed, the concentration will be within safe concentration range, and protection system can effectively provide protection for personnel.

\section{Expectations}

The analysis of simulation results and actual situation can provide ideas for further research.

(1) From the simulation results, when the fan flow starts to be the maximum, the vehicle quickly reaches the index overpressure value, and there is a difference with the actual situation where the steady state is reached after some time. The fan curve needs to be set as boundary conditions in the simulation computation in the next step.

(2) From the simulation results, the opening and closing state of the window is the main source of the distribution of the flow field in the vehicle. Before the calculation in the next step, it is necessary to simulate the vehicle specifically, and the flow field distribution obtained is more in line with the actual situation.

(3) From the simulation results, the pollutant concentration distribution in the vehicle after the fan is turned on is a short process, mainly because the fan quickly reaches the steady state, and the influence of wind speed and air leakage area on the distribution is not seen. In the next step, based on the simulation of the fan curve added, the distribution in the vehicle can be calculated by adjusting the wind speed and air leakage area in combination with the actual application of the vehicle, and then the correlation can be constructed to provide suggestions for protection.

(4) From the simulation results, it is difficult to quickly identify the specific affected area. Safe concentration and cumulative concentration can be monitored in the next simulation computation so that the hazardous area can be quickly distinguished after the post-processing to provide suggestions for protection.

\section{References}

[1] Wang Xiaodong (translate). Computational Fluid Dynamics -A Practical Approach [M]. Shenyang: Northeastern University Press, 2014

[2] Li Zhi (translate). Fluid dynamics [M]. Beijing: Higher Education Press, 2013

[3] Chen Jinzhou, Chen Haiping, Wang Xuanyu, et al. Chemical Weapon Effects and Destruction [M]. Beijing: Weapon Industry Press, 2002

[4] Zhang Jinrong. Research on Model Construction of Hazardous Gas Diffusion after Leakage in Road Transportation and Emergency Management [C]. Chang’an University, 2016:39-79

[5] Xue Haiqiang. Numerical Study on Leakage and Diffusion of Indoor Combustible Gas [C]. Shandong Jianzhu University, 2010:23-68 